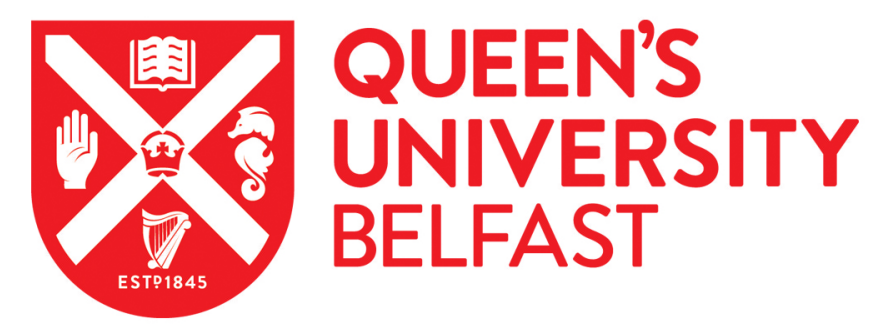

\title{
lonic liquids tethered to a preorganised 1,2-diamide motif for extraction of lanthanides
}

Gunaratne, H., Nockemann, P., Robertson, P., Stella, L., Boyd, R., \& Seddon, K. (2019). Ionic liquids tethered to a preorganised 1,2-diamide motif for extraction of lanthanides. Green Chemistry, 21, 2583-2588. https://doi.org/10.1039/C9GC00089E

\section{Published in:}

Green Chemistry

\section{Document Version:}

Peer reviewed version

\section{Queen's University Belfast - Research Portal:}

Link to publication record in Queen's University Belfast Research Portal

\section{Publisher rights}

Copyright 2019 RSC. This work is made available online in accordance with the publisher's policies. Please refer to any applicable terms of use of the publisher.

\section{General rights}

Copyright for the publications made accessible via the Queen's University Belfast Research Portal is retained by the author(s) and / or other copyright owners and it is a condition of accessing these publications that users recognise and abide by the legal requirements associated with these rights.

Take down policy

The Research Portal is Queen's institutional repository that provides access to Queen's research output. Every effort has been made to ensure that content in the Research Portal does not infringe any person's rights, or applicable UK laws. If you discover content in the Research Portal that you believe breaches copyright or violates any law, please contact openaccess@qub.ac.uk. 


\section{Green Chemistry}

\section{COMMUNICATION}

\section{lonic liquids tethered to a preorganised 1,2-diamide motif for extraction of lanthanides}

Received 00th January 20xx, Accepted 00th January 20xx

\begin{abstract}
DOI: $10.1039 / \times 0 \times x 00000 x$
\end{abstract}

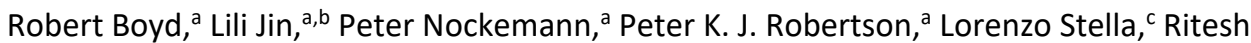
Ruhela, ${ }^{* a, d}$ Kenneth R. Seddon ${ }^{a, e}$ and H. Q. Nimal Gunaratne*a

www.rsc.org/

New preorganised 1,2-diamide functionalised, bidentate ligandembedded hydrophobic ionic liquids (DAILs) were synthesised, which enabled efficient extraction of lanthanides from an aqueous phase. The selective metal binding ability of the functionalised ionic liquid was examined by NMR, FTIR and a structure optimisation calculation. The selective liquid-liquid extraction behavior with DAILs was demonstrated.

During the past two decades, interest in developing hard-donor complexing agents that are promising extractants for the separation of lanthanides from other metals has received significant attention. ${ }^{1}$ Among the most studied cases are those containing either the phosphoryl oxygen (e.g. phosphine oxides, phosphonates, etc.), carbonyl oxygen (e.g. amides) and a mixture of both (carbamoyl phosphine oxides) as donors. ${ }^{2}$ Since the vast majority of amides remain unprotonated in acidic media, suitably substituted amide-based ligands have been a subject of extensive research as potential extractants for these metals. For example, a class of substituted malonic diamides ${ }^{3}$ and tetraalkyl-diglycolamides that were extensively examined with emphasis on the properties of tetraoctyl-diglycolamide $(\text { TODGA })^{4}$ which was utilised, diluted with a hydrocarbon, as an extractant for $\mathrm{Pu}(\mathrm{IV}), \mathrm{Np}(\mathrm{IV}), \mathrm{Am}(\mathrm{III})$, and $\mathrm{Cm}(\mathrm{III})$. The extractability of TODGA toward many other metals, and its high

aThe QUILL Research Centre, School of Chemistry and Chemical Engineering, the Queen's University of Belfast, Stranmillis Road, Belfast, Northern Ireland, BT9 5AG, UK. E-mail: n.gunaratne@qub.ac.uk

bDepartment of Organic Chemistry, China Pharmaceutical University, Nanjing, 210009, P. R. China.

'Atomistic Simulation Centre, School of Mathematics and Physics, Queen's University Belfast, University Road, Belfast, UK and School of Chemistry and Chemical Engineering, Queen's University Belfast.

dMaterials Processing \& Corrosion Engineering Division, Materials Group, Bhabha Atomic Research Centre, Mumbai-400085, India.

E-mail: riteshr@barc.gov.in

eDeceased

Electronic Supplementary Information (ESI) available: Synthesis and characterisation, mass spectroscopy, ${ }^{1} \mathrm{H}$ NMR, ${ }^{13} \mathrm{C}$ NMR spectroscopy. See DOI: 10.1039/x0xx00000x extractive capacity was demonstrated to allow its application as a promising extractant, as well. ${ }^{5}$ TODGA has been extensively evaluated for the separation of actinides(III) and lanthanides(III) from a highly radioactive PUREX raffinate in both batch and centrifugal extractor experiments. ${ }^{6}$ The use of ionic liquids for selective rare earth extraction from an aqueous phase has been reported earlier as beneficial due to their non-volatile nature, phase behaviour and high recovery rate. ${ }^{7}$

In all common diamide ligands known so far as extractants for lanthanides and actinides, the ligating unit appears to exhibit a lower degree of pre-organisation. To our knowledge, there is only one report in the literature where a ligand with an 1,2-diamide unit embedded in a six-membered ring is used to generate a complex with $\mathrm{La}^{3+}$ with other associated nitrate co-ligands. ${ }^{8}$ This cyclic structural feature would provide rigidity and pre-organisation. In this communication, we describe the synthesis of a novel 1,2-diamide-functionalised ionic liquid

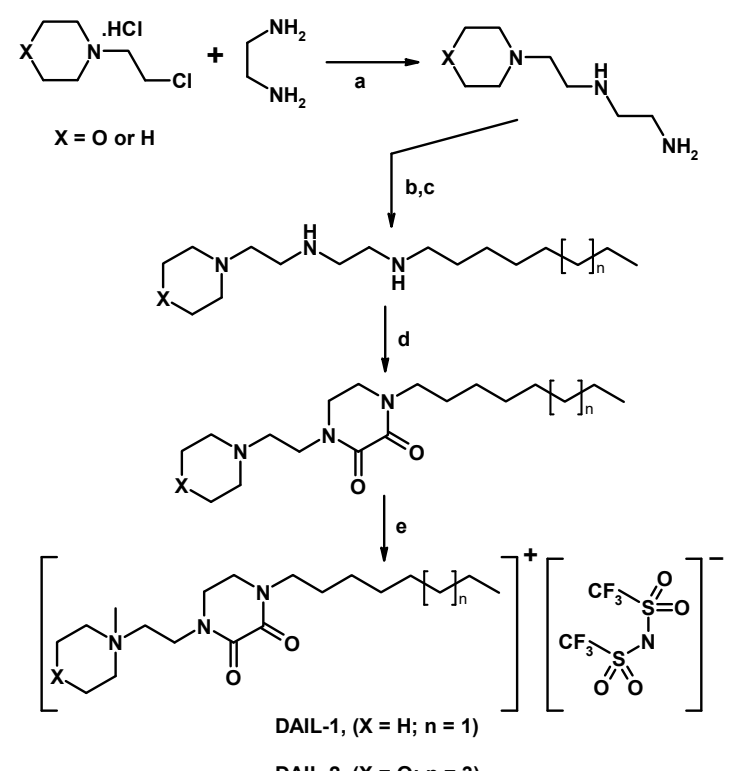

Scheme 1: Synthetic scheme for the synthesis of diamide ionic liquids (DAILs); a heated to $80^{\circ} \mathrm{C}$ for $24 \mathrm{~h}$; b treated with 1 -alkanal followed by, $\mathbf{c}, \mathrm{NaBH}_{4}$; d, heated to $80^{\circ} \mathrm{C}$ for $24 \mathrm{~h}$ with diethyl oxalate; e, treated with $\mathrm{CH}_{3}$ l followed by metathesis with Lithium bis-triflimide 
(DAILs) system where the ligating unit is placed in a rigid sixmembered ring (see Scheme 1) to be used as a recyclable hydrophobic extractant for lanthanides and actinides. The design of DAILs consists of methylmorpholinium or diethylmethylammonium cationic head groups, 2,3-pyrazolone middle unit possessing bidentate ligating ability towards rare earth metal ions and a hydrophobic decyl chain coupled with a hydrophobic, bis-triflimide anion. The inherent rigid geometry of the diamide unit embedded in DAILs is expected to display unique complexation abilities towards lanthanides while the hydrophobic tail (C8 and $\mathrm{C} 10$ ) and the associated bis-triflimide anion $\left(\mathrm{NTf}_{2}\right)$ would facilitate the extraction of such metals from an aqueous phase to a hydrophobic domain. Moreover, in comparison to flexible linear amide ligands, the ring-embedded amide structures, similar to compounds that are described here, are expected to be hydrolytically more stable at low $\mathrm{pH}$. One such pre-organised bicyclic ligand based on malondiamide which is structurally different to ours is described in the literature ${ }^{9}$ where a comparison with a linear malondiamde was made. It has to be stressed that these ligands were incorporated to another ionic liquid as additives. Here, our preorganised ligand appended ionic ligands are subjected to an examination by a liquid-liquid extraction study, NMR and FTIR investigation and a theoretical calculation showing ligand geometries around a lanthanide ion.

\section{Metal extraction studies}

Both DAIL-1 and DAIL-2 were found to be highly viscous ionic liquids and hence, to facilitate metal extraction studies, they were mixed with trihexyltetradecyl phosphonium bis-triflimide, $\left[\mathrm{P}_{6} 66_{14}\right]\left[\mathrm{NTf}_{2}\right]$, to generate a $0.005 \mathrm{M}$ solution of the active ionic liquid (binary ionic liquid). An equivolume mixture of this binary

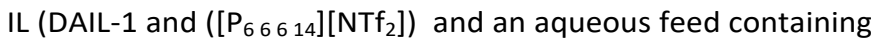
metal ions were contacted in a shaker for $30 \mathrm{~min}$. and then subsequently subjected to centrifugation to obtain two clear phases. This process was carried out at various $\mathrm{pH}$ values. The aqueous phases were separated out and analysed by ICP-OES. Figure 1 shows the variation of distribution ratio of various metal ions as a function of $\mathrm{pH}$.

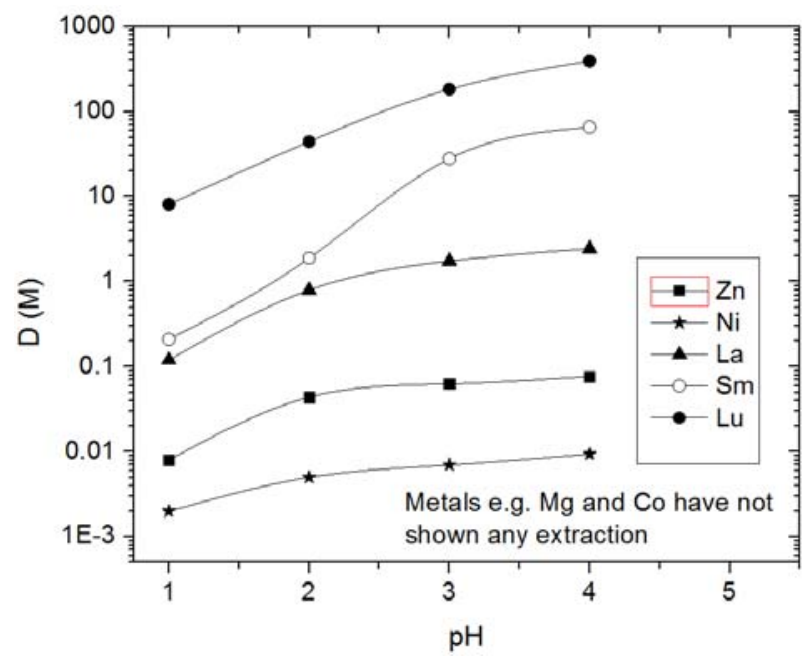

Figure 1: Distribution ratio of various metals in acidic feed solutions. $D(M)=$ $\left.[\mathrm{M}]_{\text {org./[ }} \mathrm{M}\right]_{\text {aq. }}$, organic phase $=0.005 \mathrm{M} \mathrm{DAIL-1}$ and aqueous phase $=200 \mathrm{ppm}(\mathrm{La}$, $\mathrm{Lu}, \mathrm{Sm})$ and $400 \mathrm{ppm}(\mathrm{Mg}, \mathrm{Ni}, \mathrm{Co}, \mathrm{Zn})$ dissolved in $\mathrm{HCl}$.
It can be seen that the uptake of metal ions increases with the increase in $\mathrm{pH}$ of the aqueous feed. Furthermore, high distribution ratio of rare earth metals over other transition and base metals indicated high selectivity of DAIL for rare earth metals.

As expected, on decreasing acidity of aqueous feed, an increase in the distribution ratio $\left(D_{M}\right)$ was observed for all metals extracted, as shown in Figure 1. This is due to the competition between $\mathrm{H}^{+}$and $\mathrm{M}^{\mathrm{n}}$ towards the ligand bearing ionic liquid. Metal ions such as $\mathrm{Mg}^{2+}$ and $\mathrm{Co}^{2+}$ were not extracted at all. As typical for these types of amide ligands, higher distribution ratios were associated for the extractability of heavier lanthanide ions. Very high extractability of lanthanides over other metals provides an opportunity to selectively separate the former ones. Furthermore, other co-extracted metal ions (e.g. $\mathrm{Zn}^{2+}, \mathrm{Ni}^{2+}$, etc., having D values less than 0.08; (see ESI Table S1 for distribution constants obtained for a selection of metal ions) can be easily scrubbed out with feed acid solutions leaving behind the lanthanides loaded ionic liquid phase, and thereby providing very efficient separation factors. ${ }^{10}$ The absence of softer amine donor ligands in DAIL is responsible for the lower affinity towards common $1^{\text {st }}$ row transition metal ions. ${ }^{11}$ Moreover, the hydrolytic stability of the DAILs in acidic media was investigated by NMR spectroscopy, in order to establish their longevity for reuse. In $\mathrm{CD}_{3} \mathrm{OD} / \mathrm{D}_{2} \mathrm{O}(80 / 20 \mathrm{v} / \mathrm{v})$ saturated with hydrochloric acid, DAILs showed no sign of decomposition even after a week. In general, simple linear amides are prone to slow hydrolysis in acidic media. ${ }^{12}$

A comparison was made between the performance of DAIL-1 and other diamide extractants (both non-ionic and ionic systems) reported in the literature with regards to the $D$ values. Despite having $D$ values sometimes better and at times lower, DAIL-1 shows excellent selectivity for heavier lanthanides and displays superior separation factors with respect to other systems (see ESI Table S2).

\section{Infrared investigation}

In this study, two lanthanide family metal ions, vis, $\mathrm{La}^{3+}$ and $\mathrm{Lu}^{3+}$ were treated with DAIL-1 (maintaining $\left[\mathrm{M}^{3+}\right]:[\mathrm{DAIL}]=1: 3$ ) and the complexes thus obtained were analysed by FTIR. Figure 2 shows the FT-IR spectra of DAIL, $\mathrm{La}^{3+}$-DAIL and $\mathrm{Lu}^{3+}$-DAIL complexes. The observed significant downward shift to lower wave numbers for the carbonyl group stretching frequency from $1672 \mathrm{~cm}^{-1}$ to $1648 \mathrm{~cm}^{-1}$ indicated the presence of a strong interaction of carbonyl oxygen of the amidic moiety with metal 


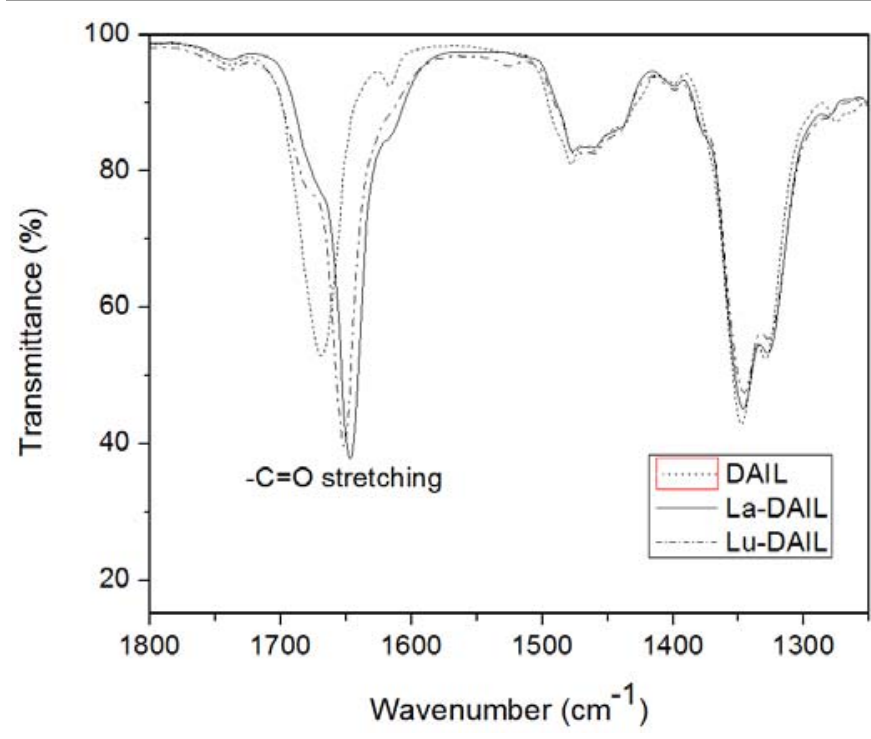

Figure 2: Infrared spectra of DAIL-1-lanthanide complexes, focused on the carbonyl stretching region. $\left[\mathrm{M}^{3+}\right]$ : $[\mathrm{DAlL}-1]=1: 3$; all samples were used in neat form without invoking any solvent.

ions. This type of La-O bond formation leads to reducing the bond order of the amide carbonyl group resulting in the changes seen in the FTIR spectra. ${ }^{13}$ This is also indicative of the presence of the significant proportion of negative charge on the carbonyl oxygen due to delocalisation of lone pair on the amide nitrogen (see below and also see ESI Scheme A for comparison with bismalonamide resonance structures).<smiles>CN1CCN(C)C(=O)C1=O</smiles>

Notably, no significant changes in the FTIRs were observed in the $1520-1300 \mathrm{~cm}^{-1}$ region. The subtle frequency changes (of $\gamma_{\mathrm{C}=0}$ ) observed for $\mathrm{La}^{3+}$-DAIL and $\mathrm{Lu}^{3+}$-DAIL can be attributed to the differences in charge densities of the two metal ions.

\section{NMR Investigation}

The metal ion complexation with DAIL-1 was examined, both qualitatively and quantitatively, by ${ }^{1} \mathrm{H}$ and ${ }^{13} \mathrm{C} \quad \mathrm{NMR}$ spectroscopy. Notably, apart from hard 'oxo' donor atoms, no soft donor groups are present in DAIL. The amide carbonyl group that is more adjacent to the cationic unit is expected to have a slightly less donor ability in comparison to the other. The stacked ${ }^{13} \mathrm{C}$ NMR spectra shown in the Figure 3 display two different signals, at $\delta 157$ and 159 (see the expanded region of the NMR), for the dissymmetric amide carbonyl groups where the down field signal $(\delta 159)$ can be attributed to the amide group that is in close proximity to the positive charge. Diamagnetic lanthanide metal, $\mathrm{La}^{3+}$ was chosen for obvious reasons for NMR examination of complexation. As expected, both carbonyl signals were shifted down-field ${ }^{14}$ after complexation with $\mathrm{La}^{3+}$. Other notable changes occurred $\sim \delta 48$ with subtle changes seen $\delta 24-30$. Similar spectral changes due to complexation with $\mathrm{La}^{3+}$ was also observed in ${ }^{1} \mathrm{H}$ NMR (see $\mathrm{ESI})$.

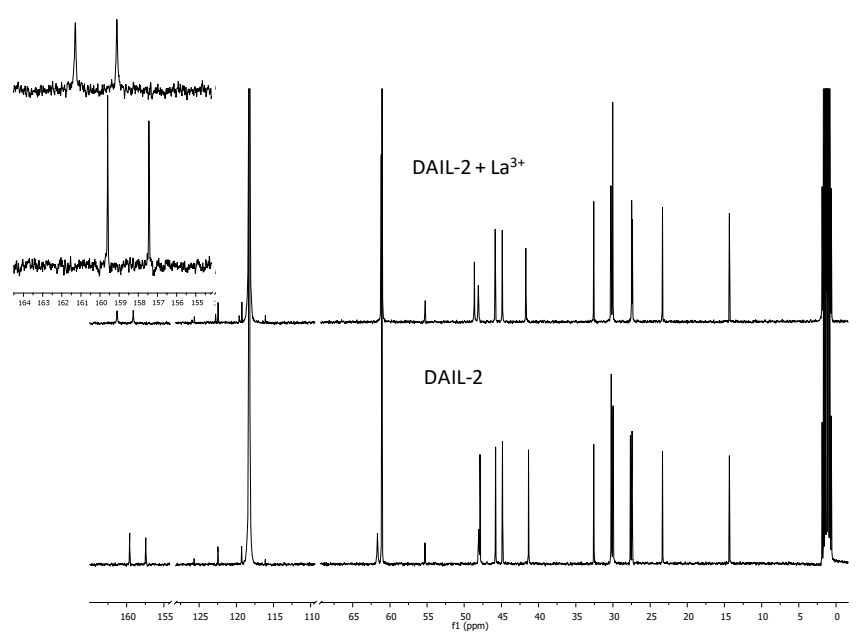

Figure 3: Stacked partial ${ }^{13} \mathrm{C}$ NMR spectra obtained with; Bottom: DAIL-2 (15 mM) and Top: an equimolar mixture DAIL-2 and $\mathrm{La}^{3+}(15 \mathrm{mM})$ in $\mathrm{CD}_{3} \mathrm{CN}$. Inset (at the top): expanded region ( $\delta 170-115)$ of the spectra showing the positions of the two amide carbonyl signals before and after complexation.

This qualitative NMR study led to carrying out a NMR titration in order to analyse the complexation process closely. The study was done with DAIL-1 and $\mathrm{LuCl}_{3}$ in $\mathrm{CD}_{3} \mathrm{OD}$ using ${ }^{1} \mathrm{H} \mathrm{NMR}$ spectrometry. Systematic changes in ${ }^{1} \mathrm{H}$ NMR that occurred due to $\mathrm{Lu}^{3+}$ complexation are shown in the stack plot depicted in Figure 4. A plot of the chemical shift changes that occur in the peak at $\delta 3.07$ due to the variation in the metal ion concentration versus the $\mathrm{Lu}^{3+}$ metal ion concentration is depicted in the Figure 5 . This graph of $\Delta \delta$ (difference in the chemical shift $=\delta_{\mathrm{M}}-\delta_{\mathrm{MO}}$ ) against $\left[\mathrm{Lu}^{3+}\right]$ exhibits a typical shape for a binding isotherm, i.e. a gradient followed by a plateau.

The metal ion concentration at the approximate position where the gradient changes to a plateau is $0.012 \mathrm{M}$ indicating the stoichiometry of the Lu ${ }^{3+}$ : DAIL-1 in the metal complex is 1:3. Even though rare earth metal ions are capable of accommodating up to ten ligating groups in their coordination sphere, the presence of a positive charge on the ligating motif of DAILs and some associated steric factors appear to have curtailed the number of DAIL-cations surrounding the metal ion. ${ }^{15} \mathrm{~A}$ closer examination of the plateau region of Figure 5 reveals that there is a slight gradient in this region which appears to suggest the difficulty in accommodating another DAIL-cation into the metal coordination sphere already having three DAIL cations. A further verification for these observations may be unearthed from a theoretical structure optimisation study. 


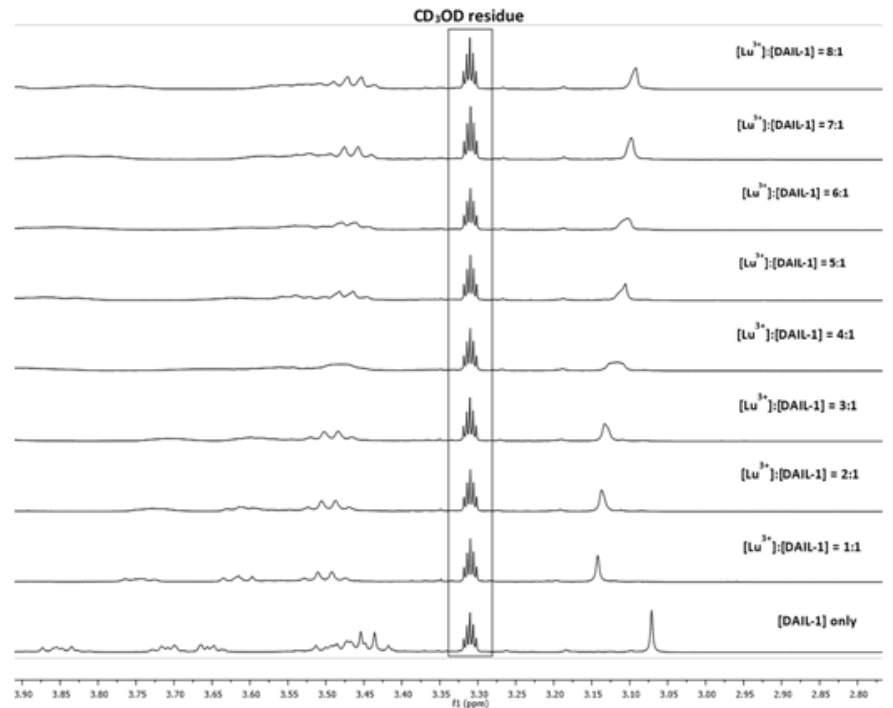

Figure 4: Stacked partial ${ }^{1} \mathrm{H}$ NMR spectra $(400 \mathrm{MHz})$ featuring the NMR titration of DAIL-1 $(0.1 \mathrm{M})$ with $\mathrm{LuCl}_{3} \cdot 6 \mathrm{H}_{2} \mathrm{O}(0.05 \mathrm{M})$ in deuterated methanol.

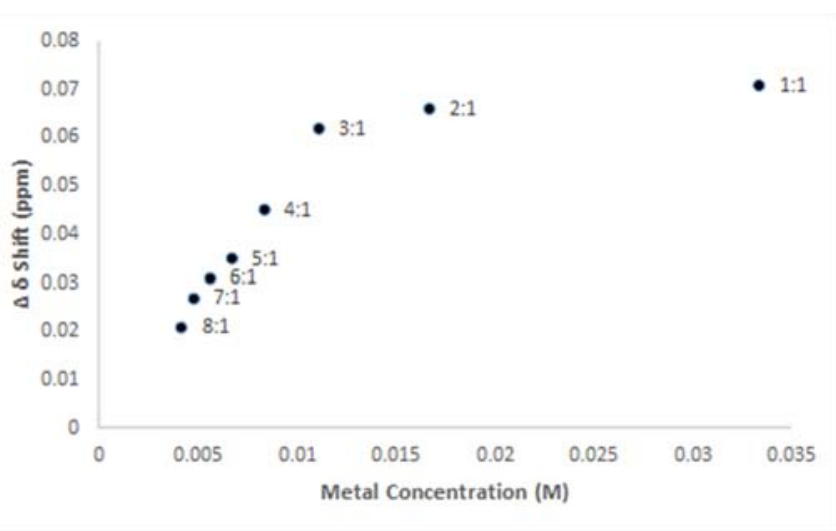

Figure 5: A plot depicting the change in shift ( $\Delta \delta$ in ppm) of the proton belonging to DAIL-1 at $3.07 \mathrm{ppm}$ on increasing the concentration of $\mathrm{LuCl}_{3} \cdot 6 \mathrm{H}_{2} \mathrm{O}$ in deuterated methanol, at $25^{\circ} \mathrm{C}$, obtained through an NMR titration. The concentration of DAIL-1 =

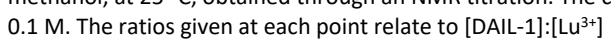

\section{Recycling study}

A recycling study of DAIL-1 was carried out with a stock solution of $\mathrm{La}$ (III) (37 ppm) which was buffered at $\mathrm{pH}=4$. Arsenazo III dye ${ }^{16}$ that is sensitive to lanthanides ions was utilised to assess the interaction of metal ion with DAIL-1 by analysing the aqueous phase post complexation. The extracting phase (DAIL-1

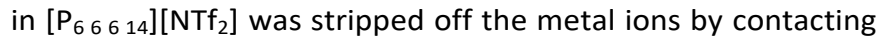
with dilute $\mathrm{HCl}(\mathrm{pH}=0.5)$ once and then the aqueous phase was analysed for $\mathrm{La}^{3+}$ using the Arsenazo III, colorimetric dye (by UVVis spectrophotometry). A second extraction of the with dilute $\mathrm{HCl}(\mathrm{pH}=0.5)$ showed that one extraction was sufficient to strip off all complexed lanthanide ions. As shown in Figure 6,

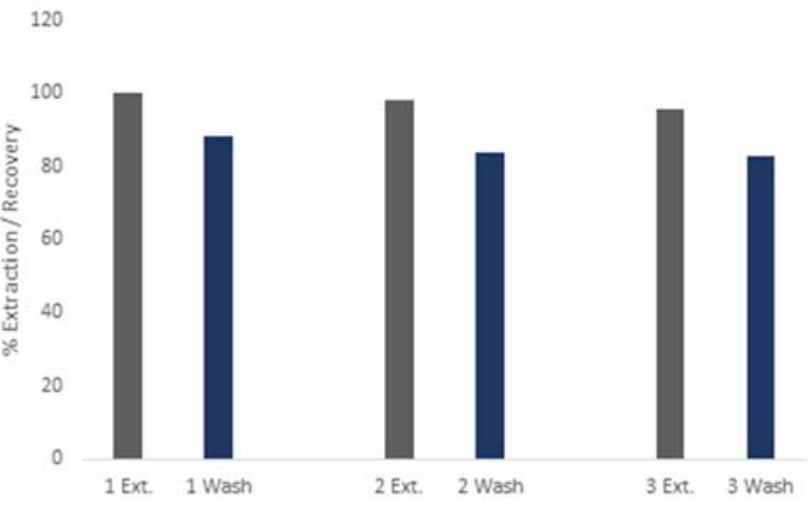

Figure 6: A bar chart showing the recycling efficiency DAIL-1 ionic liquid towards $\mathrm{La}^{3+}$ ion. This shows 3 consecutive cycles featuring an extraction step followed by stripping and $\mathrm{pH}$ adjusting steps

very good recyclability of the DAIL-1 was demonstrated with 3 recycles. Protonation of the carbonyl function in strongly acid medium is responsible for the release of the lanthanide ion. We believe, larger scale operations would bring even better recyclability of the ionic liquid ligand since the thin layer of ionic liquid on the wall of the glass vessel could not be easily recovered.

\section{Geometry Optimisation by DFT}

Finally, we undertook a theoretical study on the complexation modes of La with a simplified structural unit of DAILs. To simplify and ease the calculation, bulkier octyl or decyl groups on one amide $\mathrm{N}$ were replaced by methyl groups. The optimised geometry of such a La $^{3+}$-DAlL-2 complex is shown in Figure 7. The complex conforms to an eight-ligating coordinate system involving one bis-triflimide anion and six oxygen atoms originating from six amidic moieties of 3 DAIL cations. It is also observed that the two oxygen (in amidic moiety) atoms in a single DAIL unit form two bonds to $\mathrm{La}^{3+}$ with different bond lengths; $2.51 \AA$ and $2.72 \AA$. This is to be expected because the electron density on the amide oxygen which is adjacent to the quaternary ammonium group would be lower compared to other amide in the ring. The inductive electron withdrawal of the quaternary ammonium group is responsible for the differences in electron densities on the two amide oxygens. It should also be noted that these simulated $\mathrm{Ln}^{3+}-\mathrm{O}$ (amide) bond lengths are comparable to those observed by others using X-ray crystallography. ${ }^{17}$ 


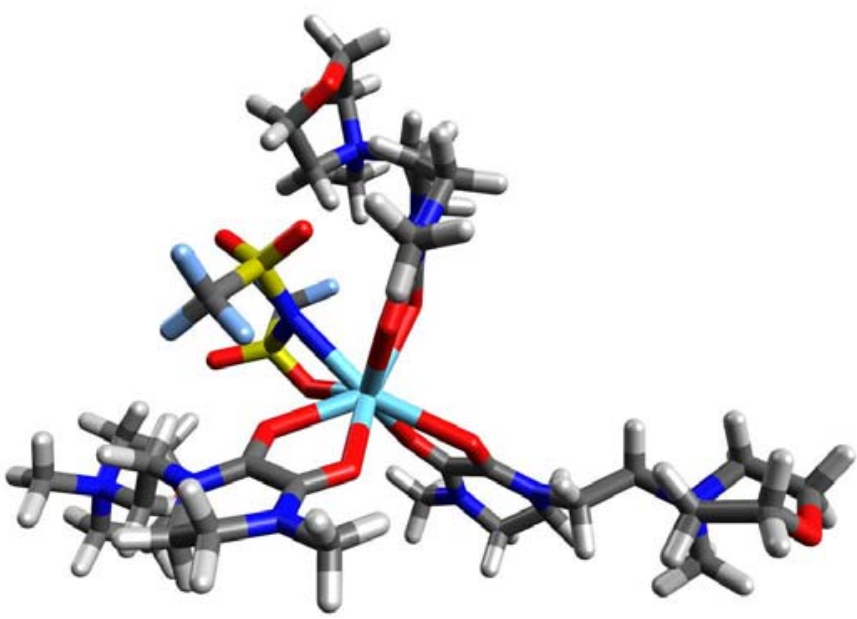

Figure 7: Optimised geometry of La $^{3+}-$ DAIL-2 complex incorporating one bistriflimide anion in the coordination sphere. ${ }^{\S}$

\section{Conclusions}

In conclusion, we have synthesised a novel ionic liquid system with an embedded 1,2-diamide structure for extracting rare earth metals from an aqueous phase containing other metal such as $\mathrm{Ni}^{2+}, \mathrm{Co}^{2+}, \mathrm{Mg}^{2+}, \mathrm{Zn}^{2+}$ and $\mathrm{Ca}^{2+}$. NMR, FTIR and extraction studies provides an insight into modes of complexation and further clarifications emanating from theoretical calculations. In general, linear amide structures are prone to slow hydrolysis in acidic media, whereas these cyclic amides are structurally robust and resist hydrolysis prolonging their life time. These functionalised ionic liquids may provide a way to process minerals containing rare earth metals.

\section{Acknowledgements}

We acknowledge the services of ASEP (School of Chemistry and Chemical Engineering) for ICP-MS analysis, Richard Murphy for NMR spectra, Conor McGrann for ES-MS spectra and Angela Brownlie for DSC and TGA analysis.

\section{Conflicts of interest}

There are no conflicts to declare.

\section{Notes and references}

$\S$ Optimization the charged (+5) $\mathrm{La}^{3+}$-DAIL-2 complex geometry was done using NWCHEM $6.6^{18}$ using the BP86 functional with Def2-SVP basis set for each element except lanthanum, for which an effective core potential (Def2-ECP) was used. Dispersion (van der Waals) interactions were also accounted for using the Grimme's D3 correction. The charged complex was subsequently micro-solvated by adding five bistriflimide anions leading to a neutral cluster. The optimisation of the neutral cluster geometry was done using MOPAC $2016^{19}$ using the sparkle/PM7 semiempirical method. ${ }^{20}$ The optimized cluster geometry was then subjected to vibrational frequency determination wherein the absence of imaginary harmonic frequencies confirmed the attainment of a local minimum of the total energy. To ease the electronic structure calculations, the bulkier alkyl groups (e.g. octyl or decyl) were replaced by methyl groups.

\section{References}

1 D. A. Atwood (ed.), The Rare Earth Elements: Fundamentals and Applications, 2012 (John Wiley \& Sons); S. A. Ansari and P.K. Mohapatra, J. Chromatography A, 2017, 1499, 1-20; F. Xie, T. A. Zhang, D. Dreisinger and F. Doyle, Minerals Engineering, 2014, 56, 10-28; J-C. G. Bünzli, J. Coord. Chem., 2014, 67, 3706-3733; C. Borra, B. Blanpain, Y. Pontikes, K. Binnemans and T. Van Gerven, J. Sustainable Metallurgy, 2016, 2, 365-386; Lucas, J., et al. Davenport, W. Rare Earths, Science, Technology, Production and Use. (Elsevier Press, 2015); Gupta, C. K. \& Krishnamurthy, N. Extractive Metallurgy of Rare Earths. (CRC Press, 2005); McLellan, B.C., et al. Sustainability of the Rare Earths Industry. Procedia Environmental Sciences, 2014, 20, 280-287; F. Xie, T. A. Zhang, D. Dreisinger and F. Doyle, Miner. Eng., 2014, 56, 10-28.

2 J. N. Mathur, M. S. Murali and K. L. Nash, Solvent Extraction and lon Exchange, 2001, 19, 357-390.

3 M. Weigl, A. Geist, K. Gompper and J. Kim, Solvent Extraction and lon Exchange, 2007, 19, 215-229.

4 S. A. Ansari, P. N. Pathak, and V. K. Manchanda, M. Husain, A. K. Prasad, and V. S. Parmar, Solvent Extraction and lon Exchange, 2005, 23, 463-479.

5 Y. Sasaki, Y. Tsubata, Y. Kitatsuji, Y. Sugo, N. Shirasu, Y. Morita and T. Kimura, Solvent Extraction and lon Exchange, 2013, 31, 401-415.

6 D. Magnusson, B. Christiansen, J. P. Glatz, R. Malmbeck, G. Modolo, D. S. Purroy and C. Sorel, Solvent Extraction and Ion Exchange, 2009, 27, 26-35.

7 T. Vander Hoogerstraete, S. Wellens, K. Verachtert and K. Binnemans, Green Chem., 2013, 15, 919; I. Billard, in Including Actinides, Elsevier, 2013, vol. 43, pp. 213-273; A. Rout and K. Binnemans, Phys. Chem. Chem. Phys., 2016, 18, 16039-16045.

8 X-Q Song, Y-W Wang, J-R Zheng, W-S Liu and M-Y Tan, Spectrochim. Acta Part A, 2007, 68, 701-704.

9 B. W. Parks, R. D. Gilbertson, J. E. Hutchison, E. R. Healey and T. J. R. Weakley, B. M. Rapko, B. P. Hay, and S. I. Sinkov, G. A. Broker and R. D. Rogers, Inorg. Chem. 2006, 45, 14981507.

10 R. Ruhela, K. K. Singh, B. S. Tomar, J.N. Sharma, M. Kumar, R. C. Hubli, A. K. Suri, Separation and Purification Technology, 2012, 99, 36-43.

11 P. Atkins, T. Overton, J. Rourke, M. Weller, F. Armstrong, Shriver and Atkins's Inorganic Chemistry, 2010.

12 Patai's Chemistry of Functional Groups: Amides, 1970 (Ed. J. Zabicky), John Wiley \& Sons Ltd.

13 O. Clement, B. M. Rapko and B. P. Hay, Coord. Chem. Rev., 1998, 170, 203-243; K. B. Girma, V. Lorenz, S. Blaurock, and F. T. Edelmann, Z. Anorg. Allg. Chem., 2005, 631, 2763-2769; H. Sigel, R. B. Martin, Chem. Rev. 1982, 82, 385.

14 T. C. Woon and D. P. Fairlie, Inorg. Chem. 1992, 31, 40694074; G. Castro, R. Bastida, A. Macías, P. Perez-Lourido, C. Platas-Iglesias, and L. Valencia, Inorg. Chem. 2015, 54, 1671-1683.

15 K. Dehnicke and A. Greiner, Angew. Chem. Int. Ed., 2003, 42, 1340-1354; J-C. G. Bünzli, J. Coord. Chem., 2014, 67, 3706-3733; E. Bodo, J. Phys. Chem. B, 2015, 119, 1183311838; X.-Z. Li, L.-P. Zhou, L.-L. Yan, Y.-M. Dong, Z-L. Bai, X-Q. Sun, J. Diwu, S. Wang, J-C. Bünzli and Q-F Sun, L. Öhrström, Nat. Chem., 2018, 10, 372. 
16 J. Uhrovcik, M. Gyevathova, J. Lesny, Nova Biotech. Chimica, 2013, 12, 93; C. Hogendoorn, P. Roszczenko-Jasinska, N. C. Martinez-Gomez, J. de Graaff, P. Grassl, A. Pol, H. J. M. Op den Camp and L. J. Daumann, Appl. Environ. Microbiol. 2018 84, e02887-17; K. L. Nash, D. Brigham, T. C. Shehee and A. Martin, Dalton Trans., 2012, 41, 14547-14556.

17 G. Castro, R. Bastida, A. Macías, P. Pérez-Lourido, C. PlatasIglesias and L. Valencia, Inorg. Chem. 2015, 54, 1671-1683.

18 M. Valiev, E. J. Bylaska, N. Govind, K. Kowalski, T. P. Straatsma, H. J. J. van Dam, D. Wang, J. Nieplocha, E. Apra, T. L. Windus, W. A. de Jong, Comput. Phys. Commun. 2010, 181, 1477

19 MOPAC2016, J. J. P. Stewart, Stewart Computational Chemistry, Colorado Springs, CO, USA, http://OpenMOPAC.net (2016).

20 J. D. L. Dutra, M. A. M. Filho, G. B. Rocha, R. O. Freire, A. M. Simas, and J. J. P. Stewart, "Sparkle/PM7 Lanthanide Parameters for the Modeling of Complexes and Materials" J. Chem.Theory and Computation, 2013, 9, 3333 